\title{
Ginkgo Biloba Extract 761 Reduces Vascular Permeability of the Ovary and Improves the Symptom of Ovarian Hyperstimulation Syndrome in a Rat Model
}

\author{
Jie Zhang \\ Dalian Municipal Women and Children's Medical Center \\ Jie Huang \\ Dalian Municipal Women and Children's Medical Center \\ Xinhuan He \\ Dalian Municipal Women and Children's Medical Center
}

Ning Li

Dalian Municipal Women and Children's Medical Center

\section{Yu Miao}

Dalian Municipal Women and Children's Medical Center

\section{Beiqing Li}

Dalian Municipal Women and Children's Medical Center

\section{Xiaoguang Shao}

Dalian Municipal Women and Children's Medical Center

Ningning Wang ( $\nabla$ zkxwnn@dmu.edu.cn )

Dalian Medical University https://orcid.org/0000-0001-7883-4556

\section{Research}

Keywords: Ginkgo biloba extract, EGb761, ovarian hyperstimulation syndrome, OHSS, vascular permeability, VEGF, VEGFR

Posted Date: January 5th, 2021

DOl: https://doi.org/10.21203/rs.3.rs-138897/v1

License: (9) (1) This work is licensed under a Creative Commons Attribution 4.0 International License. Read Full License 
Version of Record: A version of this preprint was published at Gynecological Endocrinology on December 29th, 2021. See the published version at https://doi.org/10.1080/09513590.2021.2011198. 


\section{Abstract}

\section{Background}

Ginkgo biloba extract (EGb) has been widely applied in the treatment of cerebrovascular and neurological diseases. However, the effect of EGb761 on ovarian hyperstimulation syndrome (OHSS), a vascular disorder and life-threatening complication of In vitro fertilization and Intracytoplasmic sperm injection therapy (IVF/ICSI), has not been evaluated.

\section{Methods}

Forty female Wistar rats aged 22-days old (D22) were divided into eight groups: Control rats received intraperitoneal injection of saline for 5 consecutive days (D22-D26); OHSS-model group received $10 \mathrm{IU}$ equine chorionic gonadotropin (eCG) for 4 consecutive days (D22-D25) and $30 \mathrm{IU}$ of human chorionic gonadotropin ( $\mathrm{hCG}$ ) on the 5th day (D26); Prophylactic treatment group received three doses of EGb761 $(50 \mathrm{mg} / \mathrm{kg} / \mathrm{d}, 100 \mathrm{mg} / \mathrm{kg} / \mathrm{d}, 200 \mathrm{mg} / \mathrm{kg} / \mathrm{d})$ one hour before injection of eCG (hCG) for 7 consecutive days; Therapeutic treatment group received three doses of EGb761 $(50 \mathrm{mg} / \mathrm{kg} / \mathrm{d}, 100 \mathrm{mg} / \mathrm{kg} / \mathrm{d}, 200 \mathrm{mg} / \mathrm{kg} / \mathrm{d}) 48$ hours after injection of eCG (hCG) for 7 consecutive days.

Results

We found the therapeutic treatment group exhibited the lowest ovarian and renal mass index, vascular permeability, estradiol and progesterone concentrations, vascular endothelial growth factor (VEGF) and vascular endothelial growth factor receptor (VEGFR) protein expression.

\section{Conclusions}

EGb761 decreases vascular permeability in OHSS rat model by inhibiting VEGF and VEGFR expression, which may contribute to the prevention and treatment of OHSS. Furthermore, therapeutic medication is superior to prophylactic medication.

\section{Background}

Ovarian hyperstimulation syndrome (OHSS) is a potentially life-threatening complication of In vitro fertilization and Intracytoplasmic sperm injection (IVF/ICSI) therapy [1]. OHSS is essentially a vascular disorder with clinical symptoms related to an overall increase in systemic vascular permeability. In cases of OHSS, fluid and serum proteins are constantly lost from the vasculature, leading to ascites and even progress to pleural effusion, thrombosis, and hypovolemic shock [2,3]. Studies in humans and rodents have investigated that vascular endothelial growth factor (VEGF) and its receptors VEGFR seem to play a key role in the pathological process of OHSS $[4,5]$. Moreover, these suggestions are strengthened by the findings that the occurrence of OHSS correlates with the serum concentration of VEGF, and the fact that VEGF content in the follicular fluid is frequently higher in OHSS patients than that of individuals not affected by such complication [6]. Interestingly, both the clinical pregnancy rate and abortion rate in 
OHSS patients were significantly higher than those without the syndrome. These observations suggest that early pregnancy processes, such as implantation, trophoblast invasion, and placentation, may also be affected in OHSS patients [7].

To date no specific therapy is available for OHSS, and current treatment principles include avoiding the use of human chorionic gonadotropin ( $\mathrm{hCG}$ ) to trigger oocyte maturation, postponement of embryo transfer, and intensive follow-up [3]. Obviously, these treatments are not effective in preventing development of the syndrome. The study of Zhang et al. has shown that Ginkgo biloba extract (EGb) 761 can inhibit the expression of VEGF protein and VEGF mRNA in co-cultured rat aortic endothelial cells with lysophosphatidylcholine [8]. EGb761 is standardized to contain $24 \%$ flavonoids, $6 \%$ terpenoids (ginkgolide $3.1 \%$, bilobalide $2.9 \%$ ), 5-10\% organic acid and other components. It has been extensively applied for neurological and vascular diseases, such as cerebral insufficiency, stroke, multi-infarct dementia and myocardial ischemia [9]. However, whether EGb761 is effective in preventing or treatment of OHSS has not been studied.

The aim of the present study was to investigate the possible protective effect of EGb761 on the development of OHSS and explore the underlying mechanism. With OHSS rat models, we found that EGb761 could reduce vascular permeability of the ovary, and improve the symptoms of OHSS by inhibiting the expression of VEGF and VEGFR.

\section{Methods}

\section{Animal models and interventions}

Forty female immature Wistar rats aged 22 days-old (D22) were provided by the Institute of Genome Engineered Animal Models for Human Disease of Dalian Medical University (Dalian, China). They were kept under a controlled $12 \mathrm{~h}$ light/12h dark life cycle and were fed with a standard laboratory chow diet, with free access to food and water.

The animals were randomly divided into eight groups $(n=5)$, and there was no difference in the initial body weight of each group $(P=0.382)$. The interventions of each group were conducted as below: $(1)$ CON, control rats received intraperitoneal injection of saline $(0.5 \mathrm{~mL} / 100 \mathrm{~g})$ for 5 consecutive days (D22D26); (2) OHSS, OHSS model rats received $10 \mathrm{IU}$ equine chorionic gonadotropin (eCG, Solarbio, China, $0.5 \mathrm{~mL} / 100 \mathrm{~g}$ ) for 4 consecutive days (D22-D25) and 30 IU of hCG (Ningbo Renjian Pharmaceutical Group Co.Ltd., China, $0.5 \mathrm{~mL} / 100 \mathrm{~g}$ ) on the 5th day (D26); (3) EGb761+OHSS, prophylactic treatment group received (intraperitoneal injection) three doses of EGb761 (50mg/kg/d, 100mg/kg/d, 200mg/kg/d) one hour before injection of eCG or hCG for 7 consecutive days; (4) OHSS+EGb761, therapeutic treatment group received (intraperitoneal injection) three doses of EGb761 $(50 \mathrm{mg} / \mathrm{kg} / \mathrm{d}, 100 \mathrm{mg} / \mathrm{kg} / \mathrm{d}, 200 \mathrm{mg} / \mathrm{kg} / \mathrm{d}$ ) 48 hours after injection of eCG or hCG for 7 consecutive days. Medications were stored and used according to the manufacturers' instructions. All doses are recommended to be efficacy in humans and extrapolated from animal's weight. 


\section{Measurement of peritoneal and ovarian capillary permeability}

Alterations in vascular permeability were measured by the Evans Blue (EB) dye method according to a protocol slightly modified from that described by Ujioka et al. [10]. Rats were inhalation anesthetized with diethyl ether and kept in thermal blanket to avoid hypothermia. EB dye powder (E8010, Solarbio, China) was diluted in distilled water at a final concentration of $5 \mathrm{mM}$. A fixed volume of $0.2 \mathrm{~mL}$ was administered via the caudal vein in each rat. Thirty min after the EB injection, the peritoneal cavity was irrigated with $5 \mathrm{~mL}$ of $0.9 \%$ normal saline $\left(21^{\circ} \mathrm{C}\right)$. The peritoneal irrigated fluid was collected into tubes and was centrifuged at $900 \mathrm{~g}$ for 12 minutes at room temperature. Immediately after the peritoneal irrigation, blood was obtained by puncture of the inferior vena cava for hormone assays as described below. Then, the right atrium was incised to allow the outflow of perfusate, and the left apex was slowly injected with $50 \mathrm{~mL}$ saline for systemic circulation perfusion. After that, the ovaries were removed, weighed and incubated in $65^{\circ} \mathrm{C} \mathrm{N}, \mathrm{N}$-Dimethylformamide (D112007, Aladdin, China) for 24h. EB concentrations were determined by measuring the dye absorption at $600 \mathrm{~nm}$ with a microplate reader (Spectra MR, DynexTechnologies, USA).

\section{Hormone assay}

The serum was collected from blood samples after centrifugation with $3000 \mathrm{rpm}$ for $15 \mathrm{~min}$. All samples were quick-frozen in liquid nitrogen and stored at $-80^{\circ} \mathrm{C}$ for further use. Estradiol (E2) and progesterone (PRG) levels were determined by the electrochemiluminescence assay (Roche, German) on an automatic electrochemical luminescence immunoassay system (Cobas e 601, Roche, Switzerland). The inter- and intra-assay coefficients of variation as indicated by manufactures were $13.8 \%$ and $8.5 \%$ for E2 respectively, $10.7 \%$ and $7.2 \%$ for PRG respectively.

\section{Histologyanalysis}

Hematoxylin and eosin (H\&E) staining: Ovaries and kidneys isolated from animals were fixed in $4 \%$ paraformaldehyde, dehydrated and embedded in paraffin as previously described [11]. Paraffin-embedded tissues were serially sectioned at $5 \mu \mathrm{m}$ thickness and stained with H\&E routinely for morphological observation. Three rats in each group have been randomly selected for detection of follicular development, and the images were obtained blind with the microscopy (Olympus BX63, Japan) and Image Pro Plus software (Media Cybernetics, USA).

Immunohistochemical (IHC) analysis: Paraffin-embedded ovarian and renal tissues were performed using the DAB Detection Kit (ZLI-9018, ZSGB-BIO, China) according to the manufacturer's instructions. Sections were deparaffinized, hydrated and rinsed before heat-mediated antigen retrieval in $0.01 \mathrm{M} \mathrm{pH} 6.0$ sodium citrate buffer (C1010, Solarbio, China). After quenching of endogenous peroxidases with $3 \% \mathrm{H}_{2} \mathrm{O}_{2}$, sections were incubated in nonspecific staining blockers for $15 \mathrm{~min}$ and with primary antibody against VEGF (WL00009b, Wanleibio, China) at a dilution of 1:100 at $4^{\circ} \mathrm{C}$ overnight. Sections were then incubated in biotinylated secondary antibody for $15 \mathrm{~min}$ followed by avidin and biotinylated HRP (1:1) mixed 
solution incubations for $15 \mathrm{~min}$. Finally, the sections were visualized with $D A B$ and counterstained with hematoxylin, and antigen distribution was examined under light microscope.

\section{Western blotting (WB)}

Ovary and kidney samples were homogenized and lysed in lysis buffer (KGP2100, KeyGEN BioTECH, China). Protein extract was collected and subjected to BCA protein assays (Thermo, USA). Thirty microgram of protein was separated by SDS-PAGE and subsequently transferred to PVDF membrane. Afterwards, the membranes were blotted with $10 \%$ skim milk for $1 \mathrm{~h}$ at $37^{\circ} \mathrm{C}$ and incubated with primary antibodies against VEGF (WL00009b, Wanleibio, China) and VEGFR1 (AF6204, Affinity Biosciences, USA) overnight at $4^{\circ} \mathrm{C}$. After incubation with the horseradish peroxidase-conjugated goat anti-rabbit IgG (ZB2301, ZSGB-BIO, China) for $2 \mathrm{~h}$ at room temperature, the membranes were visualized with an enhanced chemiluminescence plus kit (P10100, NCM Biotech, China) and Bio-Rad ChemiDoc MP imaging system. Band density was quantified using ImageJ software.

\section{Statistical analysis}

The data were expressed as the means \pm standard error of mean (SEM) and analyzed using the SPSS 20.0 statistical software (SPSS Inc. USA). The comparisons between groups were conducted using ANOVA followed by least-significant difference (LSD) post hoc analysis, and $P \leq 0.05$ was considered statistically significant.

\section{Results}

\section{Effect of EGb761 on ovarian and renal mass index of OHSS model}

As shown in Fig. 1 \& Fig S1, OHSS model rats exhibited significantly higher ovarian $(P \leq 0.01)$, renal $(P \leq$ $0.01)$ and liver $(P \leq 0.01)$ mass indexes (organ mass / body weight) than that of the control group, while pulmonary mass index was not affected $(P=0.089)$. Compared with the model group, in terms of ovarian and renal mass indexes, all three prophylactic treatment groups (EGb761+OHSS) showed comparable measurements, while all three therapeutic treatment group (OHSS+EGb761) showed significantly lower indexes (all $P \leq 0.01$ ). However, neither prophylactic nor therapeutic EGb treatment had any effect on the elevation of liver index in OHSS. The above results demonstrated that therapeutic EGb761 treatment was effective in reducing the mass of suffered genitourinary organs in OHSS.

\section{Effect of EGb761 on vascular permeability of OHSS model}

EB concentration of all intervention groups was distinctly lower than that of the OHSS group in both peritoneal irrigated fluid and ovary. Furthermore, EB contents in the therapeutic treatment group (OHSS+EGb761) were lower than those in the prophylactic treatment group (EGb761+OHSS), and dosedependent manner was observed in both groups (Fig. 2). Thus, both EGb761 prophylactic medication and therapeutic medication could alleviate the increase of vascular permeability in OHSS, and the prophylactic medication may have a more profound effect. 


\section{Effect of EGb761 on steroid hormonal levels of OHSS model}

In the stimulated OHSS rats, levels of serum E2 and PRG were both overtly elevated (both $P \leq 0.01$ ). Notably, all three doses of prophylactic EGb761 treatment (EGb761+OHSS) further significantly increased E2 levels, while therapeutic EGb761 treatment (OHSS + EGb761) at a dose of 200mg/ $\mathrm{kg} / \mathrm{d}$ markedly decreased serum E2 levels $(P \leq 0.05)$. Similarly, all three doses $(50,100,200 \mathrm{mg} / \mathrm{kg} / \mathrm{d})$ of EGb761 therapeutic agents significantly reduced serum PRG levels (all $P \leq 0.01$ ). This indicated that hormonal disorder in OHSS could be improved by therapeutic EGb761 treatment.

\section{Effect of EGb761 on follicular development of OHSS model}

As shown in the morphological analysis (Fig. 4), the ovary of OHSS rats was characterized by the highest corpus luteum numbers and lowest graafian follicle numbers. Compared with the OHSS group, lower corpus luteum numbers and higher follicle numbers were observed in OHSS+EGb761 groups especially in the high-dose group $(200 \mathrm{mg} / \mathrm{kg} / \mathrm{d})$. However, prophylactic medication of EGb761 appeared to have limited effect on follicular development. Hence, therapeutic EGb761 agent was effective for further development of follicles in OHSS and thus reduced the secretion of estrogen.

\section{Effect of EGb761 on VEGF and VEGFR expression in ovary and kidney of OHSS model}

By quantitative analysis of WB (Fig. 5), we found that VEGF expression was obviously increased in ovary and kidney tissues in the OHSS group (both $P \leq 0.01$ ), which was remarkably decreased in all three OHSS+EGb761 groups. Likewise, the expression of VEGFR in the ovary was significantly restrained by all three doses of EGb761 therapeutic agents, and the expression of VEGFR in the kidney was significantly suppressed by 100 and $200 \mathrm{mg} / \mathrm{kg} / \mathrm{d}$ dose of EGb761 therapeutic agents. Correspondingly, protein localization analysis with IHC (Fig. 6) also displayed a soared expression of VEGF in the kidney and ovary of the OHSS rats, which was distinctly damping after EGb761 treatment. In addition, there was no significant difference in pulmonary VEGF protein expression among the groups. However, consistent with the increased liver mass index in the OHSS group, VEGF protein was highly expressed in the liver of OHSS rats $(P \leq 0.01)$, which was observably decreased by prophylactic EGb761 therapeutic medication (Fig $S 1 \& S 2)$, indicating a certain contribution to reducing the peritoneal capillary permeability.

\section{Discussion}

In the present study, we explored the protective effects of EGb761 on OHSS by using a rat model, and the effects of prophylactic and therapeutic EGb761 treatment were compared. The observed follicular development in ovarian morphology and increases in vascular permeability suggested that the rat model mirrored the human condition. In addition, ovarian and renal mass index, E2 and PRG hormonal level, corpus luteum numbers, the protein expression of VEGF and VEGFR in the ovary and kidney were all augmented in OHSS model rats, while therapeutic EGb761 treatment could effectively improve the above performance of OHSS. 
VEGF, originally known as vascular permeability factor, is a signal protein that stimulates vasculogenesis and angiogenesis [12]. It is part of system that restores the oxygen supply to tissues when blood circulation is inadequate such as in hypoxic conditions. Current studies support a crucial role of VEGF in the OHSS development, as reviewed by Soares et al. [13], VEGF brings about increased vascular permeability by rearranging endothelial junction proteins, including cadherin and claudin-5. Using a rat model, Gómez R et al. found that both VEGF mRNA levels and vascular permeability in the mesentery and the ovaries increased following gonadotropin stimulation [14,15]. Consistently, the OHSS rat model in our study also showed a high protein expression of VEGF and VEGFR, as well as the aggravating vascular permeability in the peritoneum and ovary.

Ginkgo biloba extract, the dried leaf extract of Ginkgo ginkgo species Ginkgo with the main component flavonoids and terpene lactones, has a variety of health benefits. Ginkgo biloba extract is recognized as a strong antioxidant that can eliminate excess free radicals in the body and prevent lipid peroxidation. It has shown a high efficacy and no significant side effects in clinical application. In the early 1960s, extract of Ginkgo biloba has been applied in some countries for treatment of cerebrovascular diseases and neurological diseases. In addition, it has been considered to have immune-boosting, anti-aging effects and be beneficial for neurodegenerative disorders [16]. The study of Zhang et al. [8] has found that EGb can protect rat aortic endothelial cells from lysophosphatidylcholine by inhibiting the expression of VEGF protein and VEGF mRNA. However, the role and mechanism of EGb in OHSS has not been studied so far. Here, we suggested that EGb could decrease vascular permeability in OHSS rat model by inhibiting VEGF expression, which may contribute to the prevention and treatment of OHSS. Moreover, the therapeutic medication was more significant than prophylactic medication, providing a theoretical basis for the clinical application of EGb in the treatment of OHSS.

\section{Abbreviations}

EGb: Ginkgo biloba extract; OHSS: Ovarian hyperstimulation syndrome; IVF: In vitro fertilization; ICSI: Intracytoplasmic sperm injection; eCG: Equine chorionic gonadotropin; hCG: Human chorionic gonadotropin; VEGF: Vascular endothelial growth factor; VEGFR: Vascular endothelial growth factor receptor; EB: Evans blue; E2: Estradiol; PRG: progesterone; H\&E: Hematoxylin and eosin; IHC: Immunohistochemical; WB: Western blotting; SEM: standard error of mean; LSD: least-significant difference

\section{Declarations}

\section{Acknowledgments}

Not applicable.

\section{Authors' contributions}


Jie Zhang, Ningning Wang, Xiaoguang Shao designed the study. Jie Zhang, Jie Huang, Xinhuan He, Ning $\mathrm{Li}$, Yu Miao, Beiqing Li, Ningning Wang performed the study. Jie Zhang and Ningning Wang analyzed data, drafted and reviewed the manuscript.

\section{Funding}

Dalian Medical Science Research Project (Grant Number: 18Z1001)

\section{Availability of data and materials}

The datasets used during the current study are available from the corresponding author on reasonable request.

\section{Ethics approval}

All animal experiments were approved by Animal Ethics Committee of Dalian Medical University (Permit Number: AEE18077).

\section{Consent for publication}

Not applicable.

\section{Competing interests}

Authors do not have any conflict of interest to declare.

\section{References}

1. Nastri CO, Teixeira DM, Moroni RM, Leitão VM, Martins WP: Ovarian hyperstimulation syndrome: pathophysiology, staging, prediction and prevention. Ultrasound in obstetrics \& gynecology : the official journal of the International Society of Ultrasound in Obstetrics and Gynecology 2015, 45(4):377-393.

2. Feinberg EC: Ovarian hyperstimulation: past, present, and future. Fertility and sterility 2016, 106(6):1330.

3. Prevention and treatment of moderate and severe ovarian hyperstimulation syndrome: a guideline. Fertility and sterility 2016, 106(7):1634-1647.

4. Nouri K, Haslinger P, Szabo L, Sator M, Schreiber M, Schneeberger C, Pietrowski D: Polymorphisms of VEGF and VEGF receptors are associated with the occurrence of ovarian hyperstimulation syndrome (OHSS)-a retrospective case-control study. Journal of ovarian research 2014, 7:54.

5. Li Y, Fang L, Zhang R, Wang S, Li Y, Yan Y, Yu Y, Cheng JC, Sun YP: Melatonin stimulates VEGF expression in human granulosa-lutein cells: A potential mechanism for the pathogenesis of ovarian hyperstimulation syndrome. Molecular and cellular endocrinology 2020, 518:110981. 
6. Haas J, Bassil R, Gonen N, Meriano J, Jurisicova A, Casper RF: The VEGF and PEDF levels in the follicular fluid of patients co- treated with LETROZOLE and gonadotropins during the stimulation cycle. Reproductive biology and endocrinology : RB\&E 2018, 16(1):54.

7. Grossman LC, Michalakis KG, Browne H, Payson MD, Segars JH: The pathophysiology of ovarian hyperstimulation syndrome: an unrecognized compartment syndrome. Fertility and sterility 2010, 94(4):1392-1398.

8. Zhang L, Rui YC, Yang PY, Qiu Y, Li TJ, Liu HC: Inhibitory effects of Ginkgo biloba extract on vascular endothelial growth factor in rat aortic endothelial cells. Acta pharmacologica Sinica 2002, 23(10):919-923.

9. Tulsulkar J, Glueck B, Hinds TD, Jr., Shah ZA: Ginkgo biloba Extract Prevents Female Mice from Ischemic Brain Damage and the Mechanism Is Independent of the H01/Wnt Pathway. Translational stroke research 2016, 7(2):120-131.

10. Ujioka T, Matsuura K, Kawano T, Okamura H: Role of progesterone in capillary permeability in hyperstimulated rats. Human reproduction (Oxford, England) 1997, 12(8):1629-1634.

11. Wang N, Ma Y, Liu Z, Liu L, Yang K, Wei Y, Liu Y, Chen X, Sun X, Wen D: Hydroxytyrosol prevents $\mathrm{PM}(2.5)$-induced adiposity and insulin resistance by restraining oxidative stress related NF-KB pathway and modulation of gut microbiota in a murine model. Free radical biology \& medicine 2019, 141:393-407.

12. Itatani Y, Kawada K: Resistance to Anti-Angiogenic Therapy in Cancer-Alterations to Anti-VEGF Pathway. International journal of molecular sciences 2018, 19(4):1232.

13. Soares SR, Gómez R, Simón C, García-Velasco JA, Pellicer A: Targeting the vascular endothelial growth factor system to prevent ovarian hyperstimulation syndrome. Human reproduction update 2008, 14(4):321-333.

14. Gómez R, Simón C, Remohí J, Pellicer A: Vascular endothelial growth factor receptor-2 activation induces vascular permeability in hyperstimulated rats, and this effect is prevented by receptor blockade. Endocrinology 2002, 143(11):4339-4348.

15. Gómez R, Simón C, Remohí J, Pellicer A: Administration of moderate and high doses of gonadotropins to female rats increases ovarian vascular endothelial growth factor (VEGF) and VEGF receptor-2 expression that is associated to vascular hyperpermeability. Biology of reproduction 2003, 68(6):2164-2171.

16. Singh SK, Srivastav S, Castellani RJ, Plascencia-Villa G, Perry G: Neuroprotective and Antioxidant Effect of Ginkgo biloba Extract Against AD and Other Neurological Disorders. Neurotherapeutics : the journal of the American Society for Experimental NeuroTherapeutics 2019, 16(3):666-674.

\section{Figures}


(a)

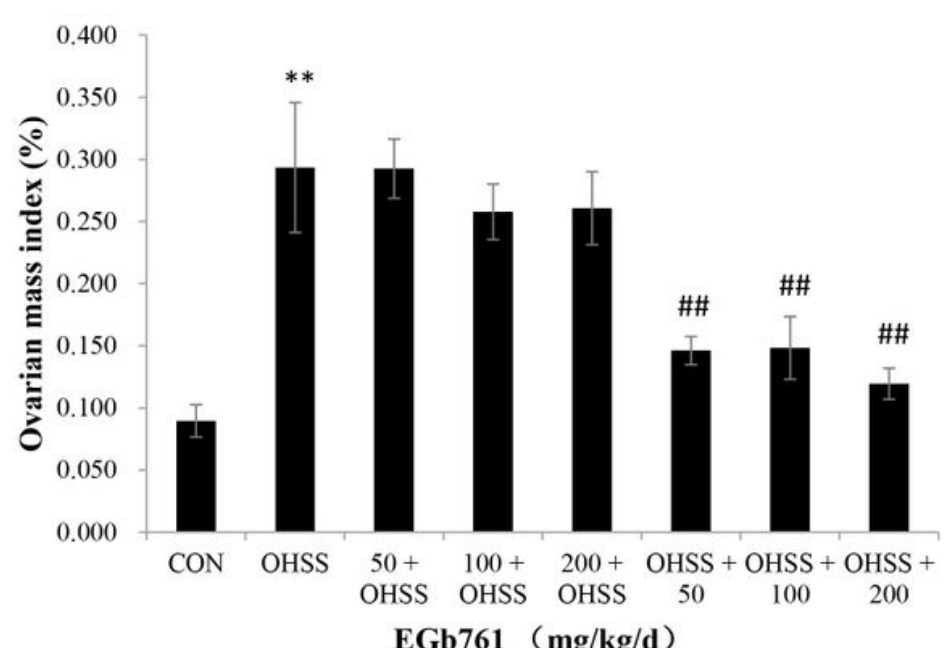

(b)

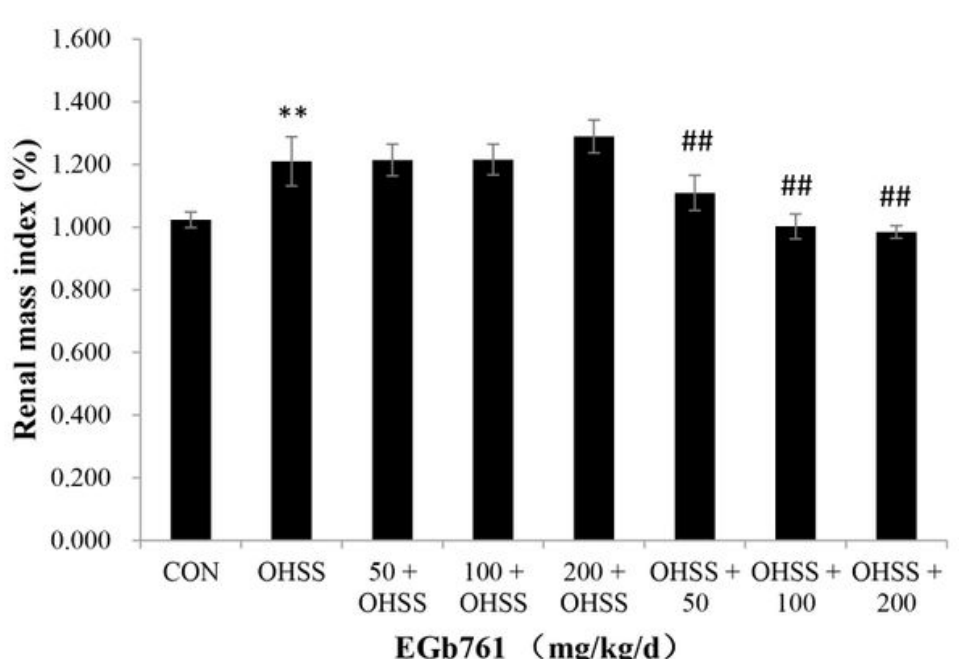

\section{Figure 1}

Effects of Ginkgo biloba extract (EGb761) on ovarian (a) and renal (b) mass index in ovarian hyperstimulation syndrome (OHSS) rats. The results are presented as means \pm SEM. Statistical analyses were conducted using one-way ANOVA followed by least-significant difference (LSD) post hoc analysis tests. For all analyses, values of $\mathrm{P} \leq 0.05$ were considered statistically significant, $\mathrm{n}=5$ per group. $* \star \mathrm{P} \leq$ 0.01 vs. CON group; \#\# $\mathrm{P} \leq 0.01$ vs. OHSS group.

(a)

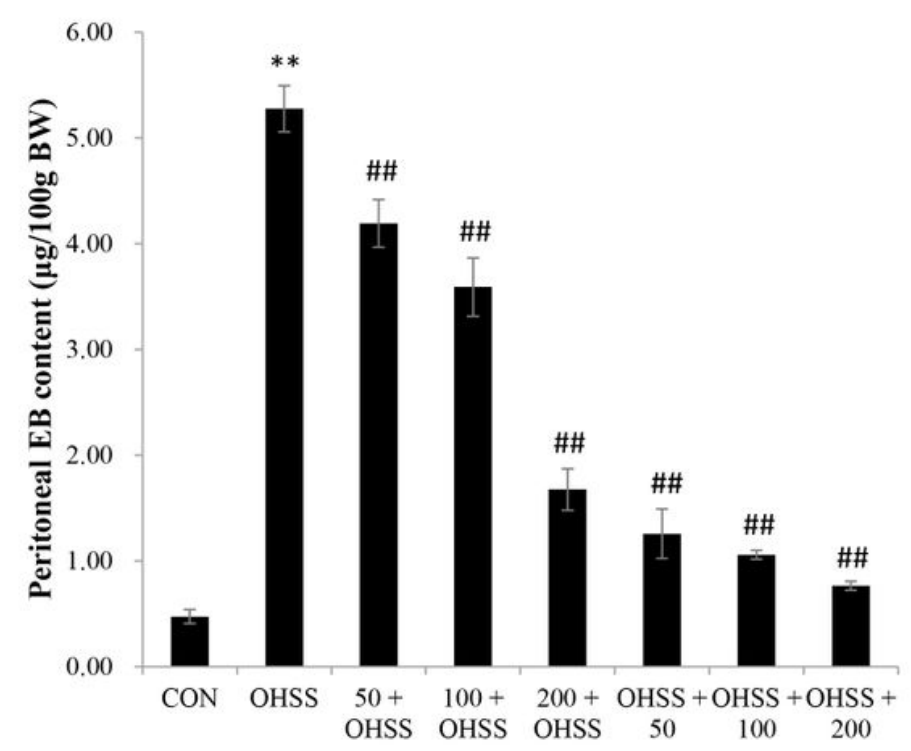

EGb761 $(\mathrm{mg} / \mathrm{kg} / \mathbf{d})$ (b)

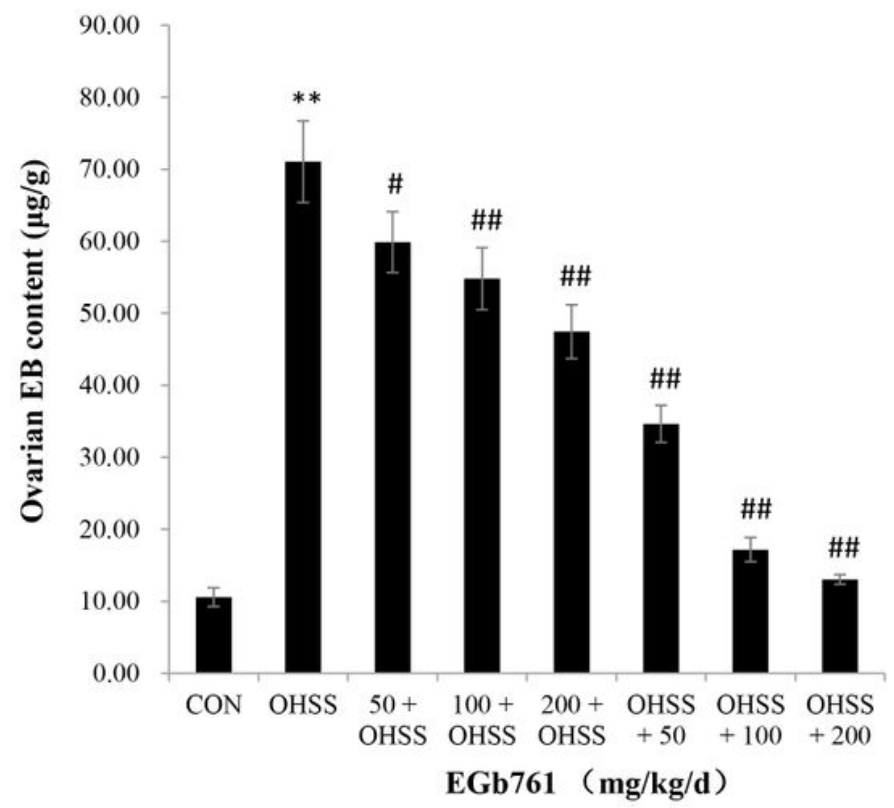

Figure 2 
Effects of Ginkgo biloba extract (EGb761) on the Evans Blue dye (EB) content of peritoneal irrigated fluid (a) and ovary (b) in ovarian hyperstimulation syndrome (OHSS) rats. EB was injected intravenously 30 min before execution. The level of EB dye in the peritoneal irrigated fluid was expressed as $\mu \mathrm{g} / 100 \mathrm{~g}$ body weight (BW), and the ovarian EB content was expressed as $\mu \mathrm{g} / \mathrm{g}$ tissue weight. The results are presented as means \pm SEM. Statistical analyses were conducted using one-way ANOVA followed by leastsignificant difference (LSD) post hoc tests. For all analyses, values of $P \leq 0.05$ were considered statistically significant, $n=3$ per group for (a) and $n=4$ per group for (b). ${ }^{\star \star} P \leq 0.01$ vs. CON group; \# $P$ $\leq 0.05, \# \# \leq 0.01$ vs. OHSS group

(a)

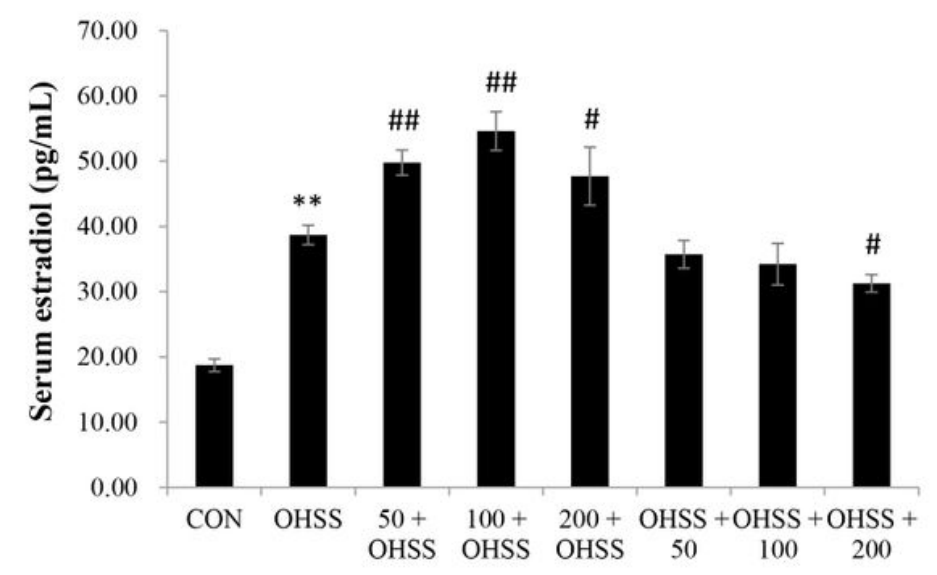

EGb761（ $\mathrm{mg} / \mathrm{kg} / \mathrm{d})$ (b)

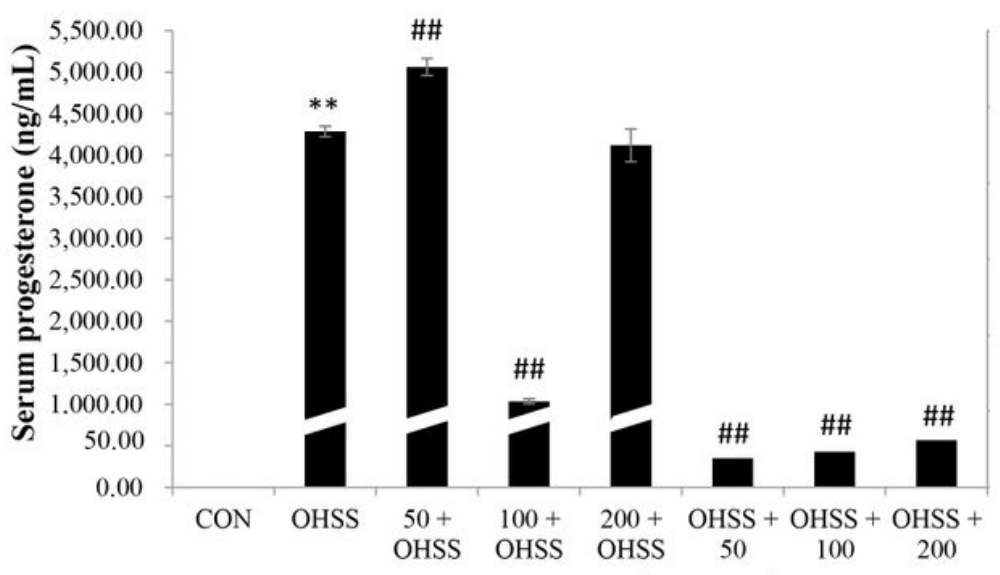

EGb761（mg/kg/d )

\section{Figure 3}

Effects of Ginkgo biloba extract (EGb761) on serum concentrations of estradiol (a) and progesterone (b) in ovarian hyperstimulation syndrome (OHSS) rats. The results are presented as means \pm SEM. Statistical analyses were conducted using one-way ANOVA followed by least-significant difference (LSD) post hoc tests. For all analyses, values of $P \leq 0.05$ were considered statistically significant, $n=5$ per group. $* \star ~ P \leq$ 0.01 vs. CON group; \# $P \leq 0.05, \# \# P \leq 0.01$ vs. OHSS group.

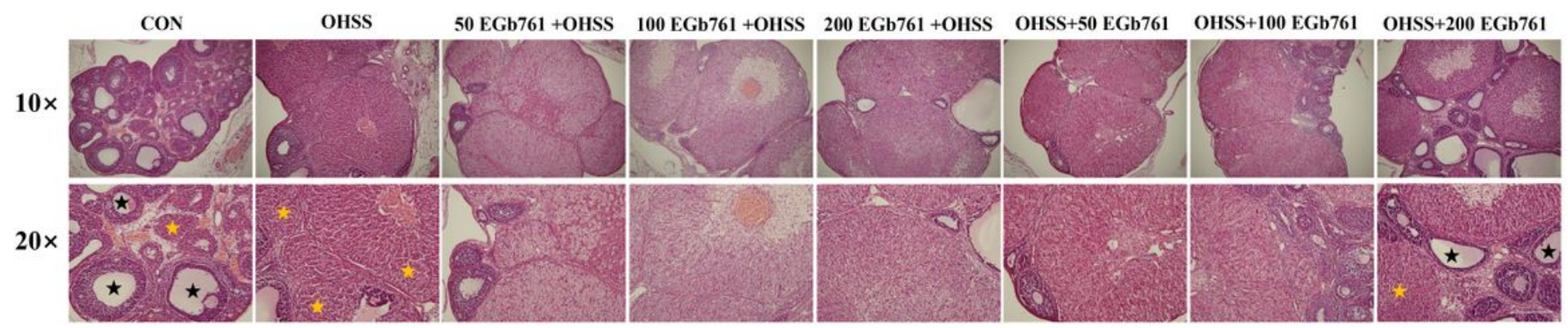

Figure 4 
Representative H\&E staining of cross-sections of ovary isolated from rats of each group. Extensive corpus luteum (yellow star) numbers and minimum graafian follicle (black star) numbers was detected in the ovary of OHSS rats, whereas lower corpus luteum numbers and higher follicle numbers were observed in OHSS+EGb761 groups especially in the high-dose group $(200 \mathrm{mg} / \mathrm{kg} / \mathrm{d})$. Images are shown at $10 x$ and $20 \times$ magnification, Scale bars: $400 \mu \mathrm{m}, \mathrm{n}=3$ per group. EGb761, Ginkgo biloba extract; OHSS, ovarian hyperstimulation syndrome

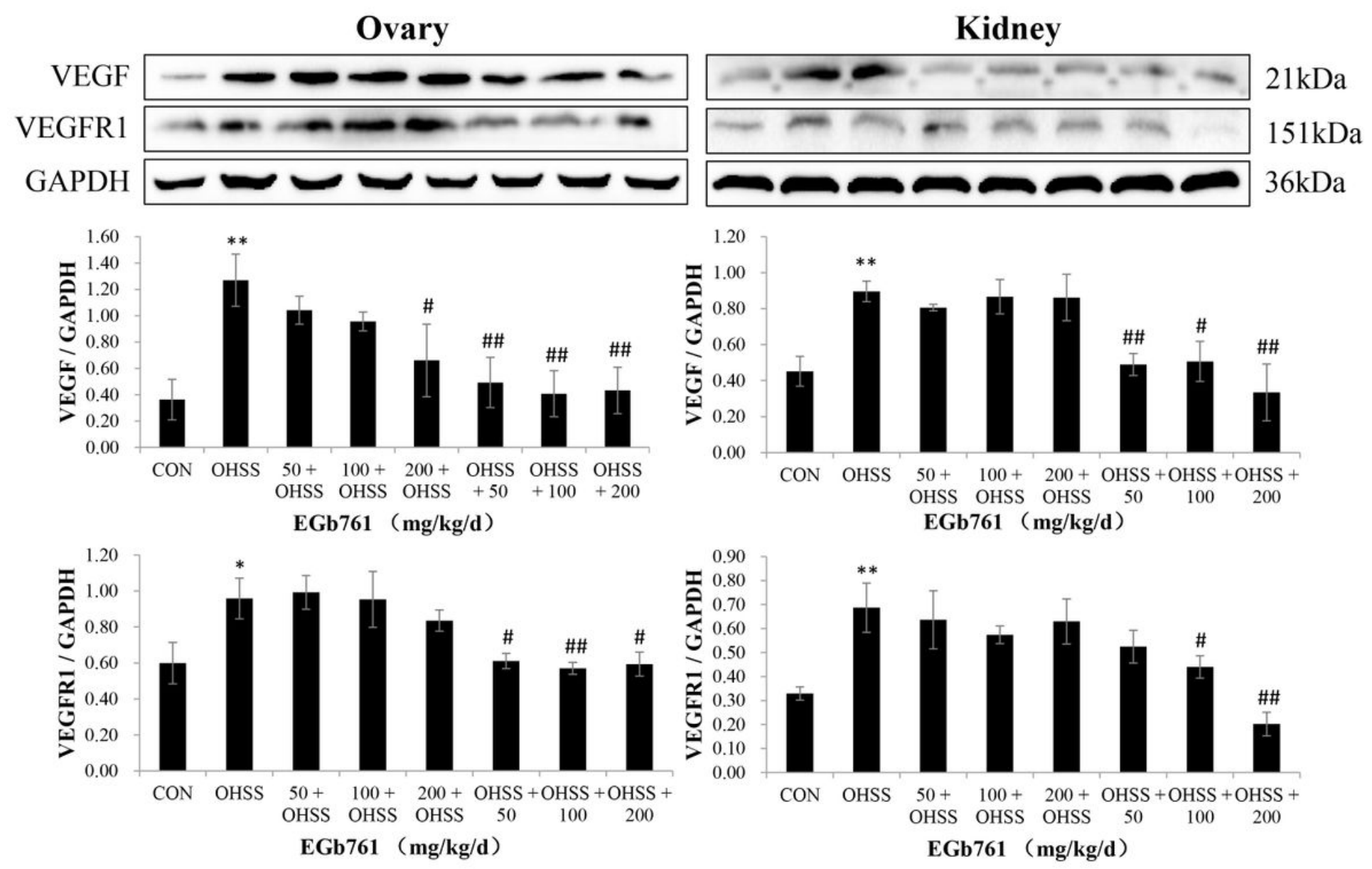

Figure 5

Western blot assays and quantification for protein expressions of vascular endothelial growth factor (VEGF), vascular endothelial growth factor receptor 1 (VEGFR1), and internal control GAPDH in the ovary and kidney. The results are presented as means \pm SEM. Statistical analyses were conducted using oneway ANOVA followed by least-significant difference (LSD) post hoc tests. For all analyses, values of $\mathrm{P} \leq$ 0.05 were considered statistically significant, $n=3$ per group. ${ }^{*} P \leq 0.01$ vs. CON group; $\# \leq 0.05$, \#\# $P$ $\leq 0.01$ vs. OHSS group. EGb761, Ginkgo biloba extract; OHSS, ovarian hyperstimulation syndrome 


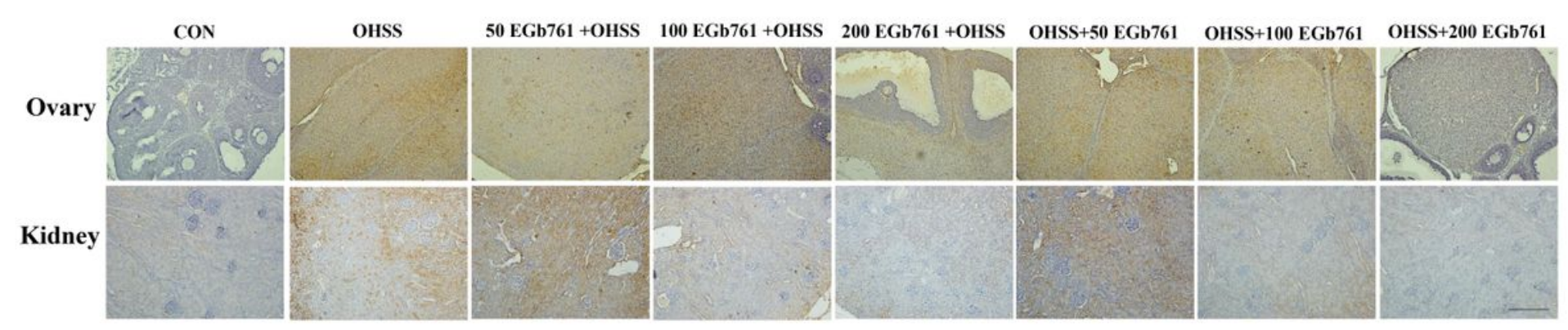

\section{Figure 6}

Immunohistochemical images of vascular endothelial growth factor (VEGF) in sections of ovary and kidney tissues. The ovarian and renal sections from ovarian hyperstimulation syndrome (OHSS) rats contained more VEGF positive cells than CON group; prophylactic treatment and therapeutic treatment of Ginkgo biloba extract (EGb761) resulted in reduced VEGF positive cells, while prophylactic treatment group showed weaker change in appearance relative to therapeutic treatment group. Images are shown at $20 \times$ magnification, scale bars: $400 \mu \mathrm{m}, \mathrm{n}=3$ per group.

\section{Supplementary Files}

This is a list of supplementary files associated with this preprint. Click to download.

- Supplementallnformation.docx 